\title{
Analysis of Consultations that are Requested from the Emergency Department
}

\author{
(1) Kemal Şener, (1) Banu Arslan, (1) Ramazan Güven, (1) Mücahit Kapçı \\ University of Health Sciences Turkey, Başakşehir Çam and Sakura City Hospital, Clinic of Emergence Medicine, \\ İstanbul, Turkey
}

\begin{abstract}
What is known on this subject?
In various studies conducted in different countries, the rate of requesting consultation in emergency services was reported to be between 20 and $56.4 \%$. It was determined that the departments where the patients were hospitalized the most were general surgery (13.8\%), neurology (13.4\%), orthopedics and traumatology (12.0\%), anesthesia (intensive care unit) (11.8\%) and pediatric surgery $(7.8 \%)$.
\end{abstract}

\begin{abstract}
What this study adds?
It is reported that approximately $19.2 \%$ of the cases were consulted in our emergency department and this rate was similar to the literature. The departments with the highest number of hospitalizations in the hospital are respectively; general surgery, internal medicine, neurology, orthopedics. It was determined that the rate of hospitalization in the emergency department was $6.2 \%$ and was lower than the rates reported in the literature.
\end{abstract}

\section{ABSTRACT}

Objective: Emergency departments (EDs) are medical units that provide healthcare to patients with diseases that have sudden onset symptoms, patients with disorders, or patients with injuries that need immediate care on a 24/7 basis. In addition to emergency patients mentioned above, EDs provide healthcare services to patients who might have an emergency medical situation later, even if their situation is not emergent initially. Emergency medicine physicians perform all resuscitative interventions to stabilize patients, identify patients who need intensive care in an undifferentiated patient pool, and provide the most appropriate treatment to make them suitable for general ward care.

Material and Methods: The current study is a retrospective and descriptive study that was conducted by analyzing the computer-based patient records of all patients who were admitted to University of Health Sciences Turkey, Bașakșehir Çam and Sakura City Hospital's Emergency Service between 09.01.2020 and 10.01.2020. All 22,459 patients who were admitted to the ED within one month were included in the study. Age and gender characteristics of the patients who received consultation, departments that made the consultation, and hospitalization rates were determined.

Results: The total number of patients who were admitted to the ED between 09.01.2020 and 10.01.2020 was 22,459 , the number of consultations was 4,290 , and the number of hospitalizations was 1,405. Of the patients for whom consultations were requested, 2,577 were male and 1,713 were female. The mean age was 45.8 years. Of the 22,459 patients who were admitted, 1,786 (7.9\%) were triaged with red tags, 9,994 (44.2\%) were triaged with yellow tags, and 10,729 (47.9\%) were triaged with green tags. The consultations were requested most frequently for orthopedics (522), pulmonology (501), and internal medicine (423). Furthermore, the list continues with general

Address for Correspondence: Kemal Şener MD, University of Health Sciences Turkey, Başakşehir Çam and Sakura City Hospital, Clinic of Emergence Medicine, İstanbul, Turkey

Phone: +90 5069156212 E-mail: drkemalsener@hotmail.com 0RCID ID: orcid.org/0000-0002-8579-6663 Received: 11.06.2021 Accepted: 25.06.2021

(C) Copyright 2021 by the Cam \& Sakura Medical Journal published by Galenos Publishing House. 


\section{ABSTRACT}

surgery (386); cardiology (335); ophthalmology (321); neurology (299); otorhinolaryngology (250); neurosurgery (204); obstetrics (138); infectious diseases (122); psychiatry (112); thoracic surgery (108); pediatric surgery (79); cardiovascular surgery (73); plastic surgery (71); anesthesiology and reanimation (140); and urology (55) departments. Of the patients for whom consultations were requested, 35.9\% were hospitalized in general wards or intensive care units, whereas $64.1 \%$ were discharged.

Conclusion: This study shows that the number of daily admissions to the emergency room is very high, and it is increasing every day. In order to not disrupt the workflow in the ED, the consultations should be responded quickly. Moreover, if possible, consultants from the high demanding departments, such as orthopedics, pulmonologist, internal diseases, general surgery, cardiology, and ophthalmology should ensure that separate doctors (doctors whose only duty would be to attend patients in their respective departments) are on call for the ED.

Keywords: Consultation, emergency service, hospitalization, internal medicine

\section{Introduction}

The emergency department (ED) is a medical unit that provides healthcare on a 24/7 basis for patients with diseases that have sudden onset symptoms, patients with disorders, or patients with injuries that need immediate care. In addition to the abovementioned emergency patients, it provides healthcare to patients who might have an emergency medical situation later, even if their situation is not emergent initially. Emergency medicine physicians perform all resuscitative interventions to stabilize patients, identify patients who need intensive care in an undifferentiated patient pool, and provide the most appropriate treatment to make them suitable for general ward care $(1,2)$.

With the increase in medical specialization and the number of specialists, there have been advances in patient management in many specialties. In cases requiring a multidisciplinary approach, physicians from various branches come together, exchange ideas, share their experiences, and collaborate to benefit patients. Therefore, consultation is an indispensable part of patient management in the ED.

The main reasons for requesting a consultation from EDs are as follows (3):

- To ensure that patients with valid indications for hospitalization are admitted to relevant clinics.

- To ask for help or advice in the diagnosis and treatment of patients.

- To organize a specific treatment or procedure for patients who require special care.

- To get approval in the discharge decision of patients with chronic diseases (such as oncology and hematology) from the $\mathrm{ED}$ (sharing the discharge responsibility).

- To arrange a detailed discharge planning and outpatient follow-up process for patients who will be discharged from the ED.

\section{Material and Methods}

This study is a retrospective and descriptive study that was conducted by analyzing the computer-based patient records of all patients who were admitted to University of Health Sciences Turkey, Başaksehir Çam and Sakura City Hospital's ED between 09.01.2020 and 10.01.2020. Approval was obtained from the Clinical Research Ethics Committee of the University of Health Sciences Turkey, Başakşehir Çam and Sakura City Hospital (protocol number: 2021-183).

In this study, the following data were obtained by retrospectively analyzing the medical forms of patients that were recorded on the Turkcell Hospital Information Management System, which is the computer-based health record system of the hospital:

- The number of patients who were admitted to the emergency room with yellow, green, and red triage tags.

- The number of patients with yellow, green, and red triage tags that were consulted.

- Department-based analysis of requested consultations.

- The number of patients that were hospitalized after consultation.

- The average age and gender distribution of the consulted patients.

All 22,459 patients who were admitted to the ED within one month were included in the study. Moreover, the age and gender characteristics of the patients who received consultation, the departments that carried out the consultation, and the rate of hospitalization were investigated.

\section{Statistical Analysis}

Statistical Package for Social Sciences (Windows 20.0) software was used for the statistical analysis of all the data obtained. All the data were summarized in the tables during 
the evaluation. Frequency tests for frequency, mean, and standard deviation values of the obtained data; MannWhitney $U$ test to compare the mean values of the obtained data, Pearson chi-square (and Fisher Exact test when necessary) to compare the non-parametric data were used. Only results with confidence intervals above $95 \%$ and $p<0.05$ were considered significant.

\section{Results}

The total number of patients who were admitted to the ED during the study period was 22,459 . The total number of consultations requested was 4,290, and the number of hospitalizations was 1,405 . Of the patients who were consulted in any department, 2,577 were male and 1,713 were female. The mean age was recorded as 45.8 years (Figure 1 ).

Of the 22,459 patients who were admitted to the ED, 1,786 (7.9\%) were triaged with red tags, 9,994 (44.2\%) with yellow tags, and 10,729 (47.9\%) with green tags (Figure 2).

Most of the patients that were admitted to the ED were seen in the green area. However, the highest numbers of consultations were requested in the yellow area. The comparison of the number of consultations with patient admissions revealed that the red area had the highest consultation rate.

The total number of consultations that were requested in this 1-month study period was 4,290. Orthopedics (522) was the most demanding medical department in terms of consultation requests, followed by pulmonology (501), and internal medicine (423). Furthermore, the list goes on with general surgery (386); cardiology (335); ophthalmology (321); neurology (299); ear, nose, and throat (250); neurosurgery (204); obstetrics (138); infectious diseases (122); psychiatry (112); thoracic surgery (108); pediatric surgery (79); cardiovascular

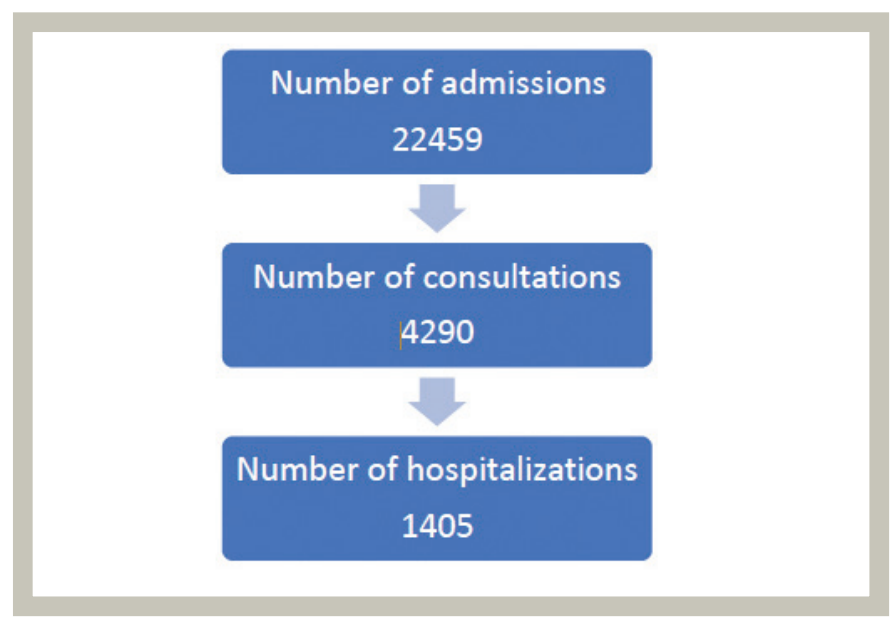

Figure 1. Flowchart surgery (73); plastic surgery (71); anesthesiology and reanimation (140); and urology (55) (Figure 3). Of the patients who were consulted in any medical department, $35.9 \%$ were hospitalized to either a hospital ward or an intensive care unit, whereas $64.1 \%$ were discharged.

When hospitalizations were assessed on a departmental basis, it was revealed that 137 patients were admitted to the general surgery department, 124 patients were admitted to the internal medicine department, 74 patients were admitted to the neurology department, and 74 patients were admitted to the orthopedics and traumatology department.

\section{Discussion}

Consultation can be vital in the management of patients in the EDs. In various studies conducted in different countries, the rate of consultation requests for emergency patients was reported to be between $20 \%$ and $56.4 \%(4,5,6,7)$. Lee et al. (8) analyzed 12 studies and discovered that the consultation rate in the EDs was between 20 and $40 \%$. Similarly, in this study, the consultation rate was found to be $19.2 \%$.

According to the study involving 32,800 patients, Köse et al. (9) stated that consultation was requested in $4.5 \%$ of all emergency clinic admissions. The consultation rates based on

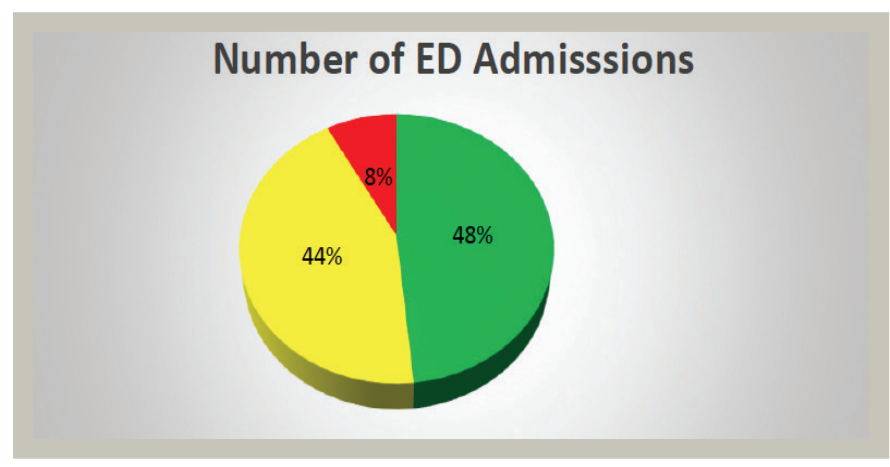

Figure 2. Number of ED admissions

ED: Emergency department

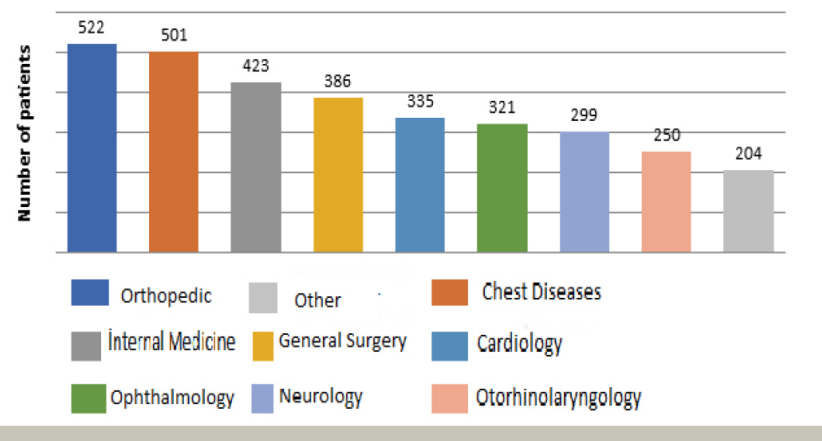

Figure 3. Number of consultations by the departments 
requested departments were reported as follows: Orthopedics (16.1\%), general surgery (15.5\%), neurology (12.5\%), internal medicine (12.2\%), and pediatric surgery (8.4\%). Similarly, in our study, orthopedic consultation was requested the most, and pulmonology consultation took the second position due to the current coronavirus disease-2019 pandemic. The reason orthopedic consultations were mostly requested could be traced to pediatric trauma patients who were also assessed in the adult emergency area.

According to the 33 studies carried out by Kılıçaslan et al. (10) in a large urban university hospital, the rate of hospitalizations from the ED was found to be approximately $12.5 \%$. In the study by Aydın et al. (11), 12.2\% of the patients admitted to the ED were hospitalized and $4.5 \%$ of them were referred to another hospital. In our study, the hospitalization rate was found to be $6.2 \%$. The low hospitalization rate can be attributed to the high number of patients with green area admissions.

In the study conducted by Köse et al. (9), the rate of hospitalization was $1.4 \%$. The departments that patients were hospitalized in the most were reported as follows: General surgery (13.8\%), neurology (13.4\%), orthopedics and traumatology $(12.0 \%)$, intensive care $(11.8 \%)$, and pediatric surgery (7.8\%). Similarly, in our study, hospitalizations from the ED were mostly in the general surgery department, followed by internal medicine, neurology, orthopedics and traumatology, intensive care, and neurosurgery departments.

When the results of our study were compared with the literature, we could conclude that our consultation rates, hospitalization rates, and the departments that emergency patients consulted with are well-matched with the current medical literature.

\section{Conclusion}

In EDs, the number of daily admissions has been increasing rapidly in the last couple of years. Therefore, overcrowding has become a serious problem causing workflow issues. In this challenging environment, the response time to consultations and the functionality of consultant physicians have become extremely important.

According to the results of this study:

1. Approximately $19.2 \%$ of the patients were consulted in our ED and this rate was well-matched with the current medical literature.
2. The patients were mostly consulted with orthopedics, pulmonology, internal medicine, and general surgery units.

3. The rate of hospitalization from the ED is 6.2\%. Even though it seems that our hospitalization rates are lesser than the rates given in the literature, the detailed analysis of patients on triage levels revealed that patients with green triage tags, which have the lowest hospitalization rates, dominated the ED admissions.

4. The departments with the highest number of hospitalizations in the hospital are; general surgery, internal medicine, neurology, and orthopedics respectively.

According to the data that were analyzed in this study, the number of emergency room admissions is very high, and this number is increasing every day. To not disrupt the workflow in the EDs, consultations should be responded to swiftly. If possible, the high demanding medical departments, such as orthopedics, pulmonologist, internal diseases, general surgery, cardiology, and ophthalmology should ensure separate doctors (doctors whose only duty would be to attend patients in their respective departments) are on call for the ED.

\section{Ethics}

Ethics Committee Approval: Approval was obtained from the Clinical Research Ethics Committee of the University of Health Sciences Turkey, Başakşehir Çam and Sakura City Hospital (protocol number: 2021-183).

Informed Consent: Retrospective study.

Peer-review: Externally peer-reviewed.

\section{Authorship Contributions}

Surgical and Medical Practices: K.Ș., B.A., R.G., M.K. Concept: K.Ş., B.A., R.G., M.K., Design: K.S.., B.A., R.G., M.K., Data Collection or Processing: K.Ş., B.A., R.G., M.K., Analysis or Interpretation: K.Ș., B.A., R.G., M.K., Literature Search: K.Ş., B.A., R.G., M.K., Writing: K.Ş., B.A., R.G., M.K.

Conflict of Interest: No conflict of interest was declared by the authors.

Financial Disclosure: The authors declared that this study received no financial support. 


\section{REFERENCES}

1. Mengert TJ. General care of the emergency department patient. Mengert TJ, Eisenberg MS, Copass MK (eds). In: Emergency Medical Therapy. 4th ed. W.B. Saunders Company, St. Louis. 1996:1-28.

2. Weiss SJ, Derlet R, Arndahl J, et al. Estimating the degree of emergency department overcrowding in academic medical centers: results of the National ED Overcrowding Study (NEDOCS). Acad Emerg Med 2004;11:38-50. Erratum in: Acad Emerg Med 2004;11:408.

3. Atilla R, Çımrın AH. Konsültasyon önerileri. In: Acil Servis ve Akademik Acil Tıp. Dokuz Eylül Üniversitesi, İzmir, 2002;184-187.

4. Geskey JM, Geeting G, West C, Hollenbeak CS. Improved physician consult response times in an academic emergency department after implementation of an institutional guideline. J Emerg Med 2013;44:999-1006.

5. Cortazzo JM, Guertler AT, Rice MM. Consultation and referral patterns from a teaching hospital emergency department. Am J Emerg Med 1993;11:456-459.

6. Vosk A. Response of consultants to the emergency department: a preliminary report. Ann Emerg Med 1998;32:574-577.
7. Brick C, Lowes J, Lovstrom L, et al. The impact of consultation on length of stay in tertiary care emergency departments. Emerg Med J 2014;31:134-138.

8. Lee RS, Woods R, Bullard M, Holroyd BR, Rowe BH. Consultations in the emergency department: a systematic review of the literature. Emerg Med J 2008;25:4-9.

9. Köse A, Köse B, Öncü MR, Tuğrul F. Admission appropriateness and profile of the patients attended to a state hospital emergency department. Gaziantep Med J 2011;17:57-62.

10. Kılıçaslan I, Bozan H, Oktay C, Göksu E. Demographic properties of patients presenting to the emergency department in Turkey. Türkiye Acil Tıp Dergisi 2005;5:5-13.

11. Aydın T, Akköse Aydın \$,, Köksal O, Özdemir F, Kulaç S, Bulut M. Evaluation of features of patients attending the Emergency Department of Uludag University Medicine Faculty Hospital and Emergency Department Practices. JAEM 2010:9:163-168. 